\title{
Uso del índice tobillo-brazo en la reclasificación del riesgo vascular en pacientes con infección VIH
}

\author{
JULIÁN OLALLA ${ }^{1}$, DANIEL SALAS², JAVIER DE LA TORRE ${ }^{1}$, \\ ALFONSO DEL ARCO ${ }^{1}$, JOSÉ LUIS PRADA ${ }^{1}$, JAVIER GARCÍA ALEGRÍA ${ }^{1}$
}

\section{Ankle-brachial index in the assessment of cardiovascular risk among HIV infected patients}

Background: Vascular risk is an important cause of morbidity and mortality in HIV infected patients. Aim: To study the value of the ankle-brachial index (ABI) in vascular risk stratification in a cohort of people with HIV infection. Patients and Methods: Vascular risk was calculated in all the patients that agreed to participate in the study and clinical reports were reviewed retrospectively. Ten year risk of fatal myocardial infarction was calculated according to Framingham equation, National Cholesterol Education Program (NCEP) III and Systematic Coronary Risk Evaluation (SCORE) project score. ABI was calculated measuring resting systolic blood pressure at the ankle, that was compared with the systolic brachial pressure. The ratio of the two pressures defined ABI, that was considered abnormal if it was $\leq 0.9$ or $\geq 1.3$. Results: A total of $231 \mathrm{HIV}$ infected patients aged 23 to 82 years (80\% males) were enrolled. Ten years risk according to Framingham equation was $8.4 \%$, 95\% confidence intervals (CI): 7.54-9.15 and according to SCORE scale was 0.8\%, 95\% CI: 0.62-1.01. According to NCEP III 9\% of patients had a high or very high cardiovascular risk. Median ABI was 1.17 (95\% CI intervals: 1.16-1.19) and 58 patients (25\%) had an abnormal value. Using ABI results, approximately $20 \%$ of patients were re-classified as having a high vascular risk. Conclusions: ABI identified approximately $20 \%$ of this cohort of HIV infected subjects as having high vascular risk.

(Rev Med Chile 2011; 139: 1039-1045).

Key words: Ankle braquial index; Cardiovascular diseases; HIV.

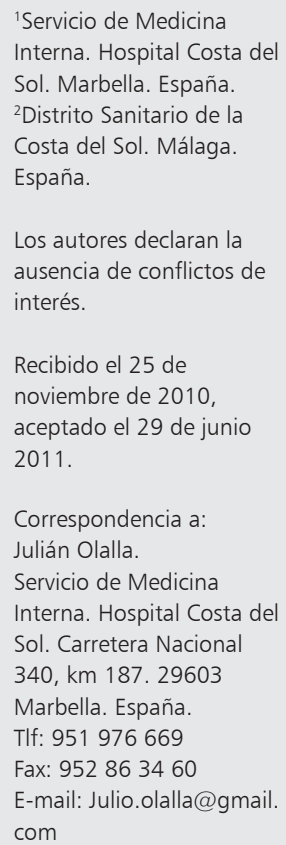

L a mayor supervivencia de los pacientes con infección por el virus de la inmunodeficiencia humana (VIH) y el progresivo envejecimiento de las cohortes, está llevando a un incremento de los eventos vasculares en este grupo de pacientes ${ }^{1}$. Tanto el propio VIH como el tratamiento antirretroviral (TAR) se han relacionado de diferentes formas con una mayor incidencia de eventos coronarios ${ }^{2,3}$. En 2007, la European AIDS Clinical Society (EACS por sus siglas en inglés) elaboró las primeras guías propias de tratamiento de los desórdenes metabólicos en pacientes con infección VIH, y recomienda el cálculo anual del riesgo vascular según la ecuación de Framingham modificada a partir de los datos del estudio D:A:D (recopilación de datos sobre efectos secundarios de los fármacos anti-VIH, en sus siglas en inglés), un estudio observacional de pacientes con infección VIH sobre efectos secundarios del TAR ${ }^{3,4}$. Además, las evidencias de estudios observacionales sobre marcadores subrogados de enfermedad vascular como el grosor íntima-media o el índice tobillobrazo (ITB), nos informan de que la afectación de estos parámetros es mayor en la población con infección por el $\mathrm{VIH}^{5,7}$. De igual forma, la propia actividad viral y el TAR pueden afectar a la elasticidad vascular ${ }^{6,8,9}$.

El hecho de que la incidencia de eventos vascu- 
lares en la población VIH sea más elevada que en la población no infectada y de que las ecuaciones actuales no predigan de forma adecuada dichos eventos, nos justifica para explorar nuevas fórmulas o marcadores subrogados de enfermedad vascular con vistas a tomar medidas de prevención más agresivas.

El ITB es una técnica simple y realizable con un entrenamiento mínimo en consultas externas. Las guías actuales ${ }^{10}$, consideran un ITB anómalo cuando éste es menor de 0,9 o mayor de 1,3 . Un metanálisis relativamente reciente, que englobaba 16 estudios con un total de 480.325 pacientes sin infección $\mathrm{VIH}^{11}$, estableció que valores de ITB $<1,1$ o $>1,4$ en varones incrementaban la incidencia de eventos coronarios.

Estudios realizados en España han mostrado una mayor prevalencia de enfermedad arterial periférica diagnosticada por ITB en población VIH respecto a la no infectada ${ }^{12}$.

Nos proponemos en este estudio evaluar la utilidad del ITB en una cohorte de pacientes con infección VIH, con vistas a determinar cuántos pacientes son reclasificados como de alto riesgo gracias al uso de esta técnica frente a las formas usuales de cálculo de dicho riesgo.

\section{Pacientes y Métodos}

Este es un estudio transversal en el que se ha calculado el riesgo de infarto agudo de miocardio a 10 años usando la ecuación de Framingham propuesta por Anderson et al en $1991^{13}$, el riesgo de evento cardiovascular mortal a 10 años usando también la detallada en el proyecto SCORE ${ }^{14}$, y la escala de clasificación de riesgo de infarto agudo de miocardio fatal o no a 10 años propuesta por el National Cholesterol Education Program (NCEP III $)^{15}$. Se consideró variable principal del estudio el valor del ITB.

La ecuación de Anderson 1991 considera a un individuo de bajo riesgo si la posibilidad de infarto agudo de miocardio a diez años, expresada en porcentaje es igual o mayor al $20 \%$, de riesgo intermedio si este porcentaje oscila entre el $10 \%$ y el $19 \%$ y de bajo riesgo por debajo de $10 \%$. El proyecto SCORE considera a un individuo de alto riesgo si el riesgo de evento cardiovascular mortal a diez años es mayor o igual a 5\%, moderado si está entre el $3-4 \%$ y bajo si es menor o igual de $2 \%$. La escala propuesta por el NCEP III considera a un individuo de alto riesgo si éste es mayor o igual al $3 \%$, intermedio en $2 \%$ y bajo menor o igual al $1 \%$. En todos los pacientes se obtuvieron datos demográficos y presencia de los factores de riesgo cardiovascular clásicos. Esta cohorte resulta de una extensión de una cohorte previa ${ }^{16}$, proveniente de pacientes de la consulta monográfica de infección VIH del Hospital Costa del Sol de Marbella, un hospital de 300 camas que atiende una población censada de en torno a 250.000 habitantes. Se propuso la participación a los diez primeros pacientes que acudieron a consulta cada día entre abril de 2007 y abril de 2009. Los pacientes no debían haber presentado eventos oportunistas en los últimos tres meses, y debían acudir de forma regular a consulta externa (al menos tres visitas anuales). El proyecto fue aprobado por el Comité Ético local y los pacientes firmaron el consentimiento informado para la realización de la prueba y la extracción de datos de su historial.

Se estableció la presencia o no de síndrome metabólico de acuerdo con los criterios de la Federación Internacional de Diabetes publicados en 2006 , esto es, circunferencia de cintura $\geq 94 \mathrm{~cm}$ en varones $\mathrm{o} \geq 80 \mathrm{~cm}$ en mujeres, y al menos dos de las siguientes condiciones: tensión arterial $\geq 135 / 85$ $\mathrm{mmHg}$, glucemia en ayunas $\geq 100 \mathrm{mg} / \mathrm{dl}$, triglicéridos $150 \mathrm{mg} / \mathrm{dl}$ o colesterol ligado a lipoproteínas de alta densidad menor de $40 \mathrm{mg} / \mathrm{dl}$ en varones o de $50 \mathrm{mg} / \mathrm{dl}$ en mujeres. Se consideró el consumo de tabaco de forma dicotómica (sí/no). Para la definición de diabetes se siguieron los criterios de la Federación Internacional de Diabetes publicados en 2005, o la toma de antidiabéticos orales o el uso de insulina en el momento del estudio. Se consignó la presencia de eventos vasculares personales o familiares si en la historia clínica del paciente se refería la presencia de ictus, cardiopatía isquémica o enfermedad arterial periférica. Como antecedentes familiares precoces se consignaron los eventos ictus, cardiopatía isquémica, enfermedad arterial periférica o muerte súbita en familiares de primer grado varones de menos de 55 años o mujeres de menos de 65.

El ITB fue considerado anormal si era $\leq 0,9$ o $\geq 1,3^{10}$. Se consideró a un paciente hipertenso si usaba medicación antihipertensiva y si las cifras de tensión arterial estaban elevadas ( $>$ 140/90 $\mathrm{mmHg}$ ) en al menos dos de las últimas tres visitas a consulta externa antes de la realización del ITB. El 
mismo tipo de criterio se siguió con la dislipemia, tomando como puntos de corte para definirla, caso de no estar tomando drogas hipolipemiantes un colesterol total de $250 \mathrm{mg} / \mathrm{dl}$ en ayunas de 12 horas.

El ITB se realizó con un ecoDoppler (multi/ super Dopplex MD2/SD2, Huntleigh Healthcare), un esfigmomanómetro y un manguito de presión. El paciente permanecía tumbado en una camilla al menos cinco minutos en una consulta tranquila siguiendo las recomendaciones estándar para la realización de la prueba ${ }^{17}$. Un solo explorador, específicamente entrenado, realizó las mediciones. No se realizaron pruebas de imagen de forma posterior al ITB. No se calculó el tamaño de muestra necesario para ninguna potencia estadística predeterminada, ni se establecieron los coeficientes de variabilidad intra e interprueba.

Se estableció qué porcentaje de pacientes que tenían un riesgo bajo o intermedio eran reclasificados a alto riesgo al presentar un ITB patológico.

De cada variable se calculó la media (en las cuantitativas) y el porcentaje (en las cualitativas). Se procedió a la comparación de variables cuantitativas normales con la función $t$ de Student, y con la Z de Wilcoxon cuando la distribución no era normal. Para la comparación de proporciones se usó la $\chi^{2}$.

\section{Resultados}

Un total de 231 pacientes participaron en el estudio. La media de edad fue de 44,44 años (Rango: $23-82$ ), siendo $80 \%$ varones. Las principales características de la serie, se resumen en la Tabla 1 . En torno al $14 \%$ de los pacientes usaban fármacos hipolipemiantes en el momento del estudio, y $8,2 \%$ medicación antihipertensiva. Trece coma nueve por ciento presentaban un índice de masa corporal mayor de $29 \mathrm{~kg} / \mathrm{m}^{2}$.

El principal grupo de transmisión del VIH fue el de contacto heterosexuales (35,1\%), seguido por homosexuales y adictos a drogas por vía parenteral y $(28,1 \%$ y $27,7 \%$, respectivamente). Cumplían criterio SIDA 108 pacientes (46,8\%).

El valor medio del ITB en esta cohorte fue de 1,17 (95\% CI: 1,16-1,19), presentando 25\% de los pacientes valores anormales.

Siguiendo los criterios de la Federación Internacional de Diabetes de 2005, 24,7\% de esta cohorte presentaba síndrome metabólico. Seis pacientes presentaban antecedentes personales de eventos vasculares mayores (tres de ellos un ictus isquémico y otros tres un síndrome coronario agudo sin elevación del ST). Tan sólo 56 pacientes aportaban un electrocardiograma o ecografía transtorácica, de los que 10 mostraban criterios de hipertrofia ventricular izquierda $(4,3 \%$ del total de pacientes). El valor medio del riesgo de evento vascular mortal a diez años dado por el SCORE fue de 0,81\% (IC95\%: 0,62-1,01). Cinco coma dos por ciento de los pacientes presentaban un riesgo alto $(\geq 5)$ atendiendo al proyecto SCORE. De éstos, 5 $(41,7 \%)$ presentaban un ITB anómalo. El valor medio del riesgo de infarto agudo de miocardio a diez años calculado por la ecuación de Anderson 1991 fue de 8,35\% (IC95\%: 7,54-9,15). El 40,8\% de los pacientes presentaban un riesgo al menos moderado $(>10 \%)$, y el $6,5 \%$ alto $(n=15)$, esto es, mayor o igual a $20 \%$. De estos últimos, sólo 8 presentaban un valor anómalo de ITB $(53,3 \%)$. En la Tabla 2 se refleja la proporción de pacientes en cada estrato de riesgo según la escala utilizada para su clasificación.

De acuerdo a los criterios propuestos en el NCEP III, 25,6\% de los pacientes presentaban un riesgo al menos moderado y $9,1 \%$ alto o muy alto. De los 23 pacientes con riesgo alto o muy alto, $10(43,5 \%)$ presentaron una ITB anómalo.

Como hemos visto, en esta cohorte de pacientes el riesgo vascular estimado por diferentes fórmulas fue, en general, bajo. Sin embargo, cuando se usó el ITB, el porcentaje de pacientes etiquetados como de alto riesgo creció. Con respecto al SCORE, el ITB reclasificó como de alto riesgo vascular a 53 pacientes $(23,1 \%$ del total), con respecto a la ecuación de Anderson 1991 a 50 pacientes $(21,7 \%)$ y con respecto al NCEP III a 48 pacientes $(20,9 \%)$. Por tanto, los pacientes clasificados como de alto riesgo vascular aunando las ecuaciones referidas y el ITB serían 28,3\% para el uso de SCORE+ITB, 28,2\% para Anderson $1991+$ ITB y $30 \%$ para el uso de NCEP III+ITB. La mayor parte de estos pacientes provienen de los clasificados como riesgo bajo antes de la realización del ITB. Setenta y cinco porciento de los pacientes con ITB alterado eran clasificados como de bajo riesgo por el proyecto SCORE, 53,5\% por la ecuación de Anderson 1991 y 68,9\% por el NCEP III. La prevalencia de los factores de riesgo cardiovascular clásicos según el valor del ITB se expone en la Tabla 3. 
Tabla 1. Prevalencia de los factores de riesgo cardiovascular clásicos en los participantes en el estudio (\%)

\begin{tabular}{|c|c|}
\hline Características basales & Valor \\
\hline Edad (años) & $44,4(43,22-45,56)$ \\
\hline Varones n (\%) & $185(80,1)$ \\
\hline $\begin{array}{l}\text { Eventos vasculares previos: } \\
\text { Cardíaco } \\
\text { Cerebral } \\
\text { Vascular periférico }\end{array}$ & $\begin{array}{l}5 \\
2 \\
3 \\
0\end{array}$ \\
\hline Duración infección VIH en años & $8,92(8,03-9,81)$ \\
\hline Caso Sida, n (\%) & $108(46,8)$ \\
\hline Peso $(\mathrm{kg})$ & $73,74(72,00-75,47)$ \\
\hline Índice de masa corporal $\left(\mathrm{kg} / \mathrm{m}^{2}\right)$ & $24,90(24,39-25,41)$ \\
\hline Presión arterial sistólica $(\mathrm{mmHg})$ & $125,76(123,52-128,01)$ \\
\hline Presión arterial diastólica $(\mathrm{mmHg})$ & $75,27(73,70-76,85)$ \\
\hline Filtrado glomerular $<60 \mathrm{ml} / \mathrm{min}, \mathrm{n}(\%)$ & $7(3)$ \\
\hline Microalbuminuria & $9(3,8)$ \\
\hline Diabetes n (\%) & $10(4,3)$ \\
\hline Hipertensión arterial n (\%) & $21(9,1)$ \\
\hline Hipertrofia ventricular izquierda n (\%) & $10(4,3)$ \\
\hline Fumador/ex fumador n (\%) & $130 / 3(56,3 / 1,3)$ \\
\hline Dislipemia n (\%) & $45(19,5)$ \\
\hline Dislipemia de acuerdo a los criterios ATP III n (\%) & $56(24,2)$ \\
\hline Historia familiar de eventos vasculares precoces n (\%) & $16(6,9)$ \\
\hline Síndrome metabólico n (\%) & $57(24,7)$ \\
\hline $\begin{array}{l}\text { Tratamiento antirretroviral actual, n (\%): } \\
\text { Análogos de nucleósido/nucleótido } \\
\text { No análogos de nucleósido } \\
\text { Inhibidores de la fusión } \\
\text { Inhibidores de la integrasa } \\
\text { Bloqueadores del correceptor CCR5 } \\
\text { Uso de Inhibidores de la proteasa }\end{array}$ & $\begin{array}{c}198(85,7) \\
120(51,9) \\
2(0,9) \\
3(1,3) \\
1(0,4) \\
86(37,2)\end{array}$ \\
\hline Glucemia (mg/dl) & $101,50(97,84-105,12)$ \\
\hline Colesterol (mg/dl) & $190,39(183,12-197,65)$ \\
\hline LDL colesterol (mg/dl) & $110,14(105,36-114,93)$ \\
\hline HDL colesterol (mg/dl) & $47,95(45,82-50,08)$ \\
\hline Triglicéridos (mg/dl) & $155,38(141,27-169,48)$ \\
\hline Creatinina (mg/dl) & $0,95(0,92-0,98)$ \\
\hline Albúmina (g/dl) & $4,38(4,31-4,45)$ \\
\hline
\end{tabular}

Para las variables cuantitativas se expresa el valor del parámetro con la media y su Intervalo de Confianza de $95 \%$. 
ITB y reclasificación del RV en VIH - J. Olalla et al

\section{Discusión}

Nuestra serie muestra por tanto, una elevada prevalencia de pacientes VIH con ITB elevado en pacientes clasificados de forma previa como de bajo riesgo por las fórmulas tradicionales. Contamos con la evidencia de que, al menos la ecuación de Anderson 1991, infraestima el riesgo vascular en pacientes con infección VIH que reciben $\mathrm{TAR}^{18}$. Este hecho justifica la búsqueda de nuevos marcadores de riesgo vascular. En nuestra serie es llamativo el efecto de reclasificación de los pacientes cuando se añade a su evaluación la práctica del ITB, ampliando hasta cerca de $30 \%$ el grupo de pacientes que serían objeto de manejo de factores de riesgo vascular de una forma más agresiva. Si observamos la clasificación previa a la realización del ITB, observamos que precisamente éste aporta más en los pacientes previamente clasificados como de bajo riesgo por las ecuaciones tradicionales.

El reclutamiento de pacientes fue ostensiblemente menor a partir de julio de 2007 sin causa aparente, derivado tan sólo de un mayor número

Tabla 2. Estratificación del RV de los pacientes con las diferentes escalas utilizadas

\begin{tabular}{|lccc|}
\hline & Bajo & Intermedio & Alto \\
\hline Anderson 1991 & 136 & 80 & 15 \\
\hline SCORE & 209 & 10 & 12 \\
\hline NCEP III & 172 & 36 & 23 \\
\hline
\end{tabular}

Indicar que se muestra estratificación de pacientes según número de ellos. de negativas de los pacientes a colaborar. En la actualidad se sigue en nuestra Unidad a unos quinientos cincuenta pacientes, por lo que la cohorte estudiada supone un cuarenta y dos por ciento.

Series españolas previas han señalado ya el aumento de la prevalencia de ITB $\leq 0,9$ en pacientes con síndrome metabólico, entre 10,1\% y 27,7\%, mostrando su valor para identificar sujetos de alto riesgo vascular ${ }^{19,20}$.

Entre los pacientes VIH, el ITB alterado se ha relacionado con el uso de inhibidores de la proteasa en al menos un estudio ${ }^{16}$, algunos de estos fármacos se han relacionado con una mayor incidencia de eventos vasculares.

En nuestro estudio hemos tomado como referencia de valores de ITB alterado 0,9 y 1,3 . Se acepta el valor de ITB $\leq 0,9$ como diagnóstico de enfermedad arterial periférica, y por tanto, como presencia de un evento vascular. Sin embargo, existe discusión sobre el límite de 1,3. Se ha aducido de forma clásica la calcificación de la pared arterial como causa de un ITB $\geq 1,3$, expresando una rigidez arterial patológica, y el hecho es que un ITB elevado en población general (en concreto mayor de 1,4$)$ se ha relacionado con una mortalidad global aumentada (HR para hombres 1,38, IC95\%: 1,17-1,62, HR para mujeres 1,23, IC95\%: $1,00-1,52)$. Estos hallazgos descritos en un metanálisis que englobaba 480.325 pacientes-año establecía también una mayor mortalidad en varones y mujeres con un ITB $\leq 1,1$ (HR 1,22 para hombres, IC95\%: 1,13-1,32, HR para mujeres 1,11, IC95\%: $1,02-1,21)$. Por tanto, más que para el diagnóstico de la enfermedad arterial periférica, nuestro afán es poder identificar pacientes subsidiarios de me-

Tabla 3. Prevalencia de los factores de riesgo cardiovascular clásicos según el valor del ITB (\%)

\begin{tabular}{|c|c|c|c|c|}
\hline Factor de riesgo cardiovascular & $\begin{array}{c}\text { ITB }<0,9 \\
n=3 \\
n \quad(\%)\end{array}$ & $\begin{array}{c}\text { ITB Normal } \\
n=173 \\
n \quad(\%)\end{array}$ & $\begin{array}{c}\text { ITB } \geq 1,3 \\
n=55 \\
n \quad(\%)\end{array}$ & $\mathbf{p}$ \\
\hline Diabetes & $1(33,3)$ & $5(2,9)$ & $4(7,3)$ & 0,01 \\
\hline Hipertensión arterial & $2(66,7)$ & $10(5,8)$ & $8(14,5)$ & $<0,0005$ \\
\hline Fumador & $2(66,7)$ & $100(58,1)$ & $28(51,9)$ & NS \\
\hline Dislipemia & $3(100)$ & $27(15,7)$ & $14(25,5)$ & $<0,0005$ \\
\hline Dislipemia de acuerdo a los criterios ATP III & $3(100)$ & $37(21,5)$ & $15(27,3)$ & 0,001 \\
\hline Síndrome metabólico & $2(66,7)$ & $39(22,7)$ & $15(27,3)$ & NS \\
\hline Historia familiar de eventos vasculares precoces & $1(33,3)$ & $11(6,4)$ & $4(7,3)$ & NS \\
\hline
\end{tabular}


didas de prevención más agresivas que aquéllos que identifican las ecuaciones clásicas ${ }^{11}$.

En este momento parece evidente que la incidencia de eventos coronarios en pacientes con infección VIH es mayor que en la población no infectada, con una Odds Ratio que oscila entre 1,5 y 2 , en comparación con la población general ${ }^{21}$. La prevalencia de ITB alterado en pacientes con infección VIH es ostensiblemente mayor en las diferentes series que la publicada para la población no infectada ${ }^{7}$, sobre todo a expensas de ITB $>1,3$. Un estudio español intentó relacionar el ITB de más de 1,4 con un aumento del grosor íntimamedia sin encontrar relación ${ }^{22}$. En la patogenia del daño coronario en el paciente con infección VIH es posible que el daño de la elasticidad vascular juegue un importante papel ${ }^{6}$, a través de fenómenos proinflamatorios y del daño endotelial ${ }^{8,9}$. Este hecho sería compatible con una importante prevalencia de ITB elevado sin una asociación clara con un grosor íntima-media elevado. El propio TAR se ha relacionado con un aumento de los eventos coronarios en amplios estudios de cohortes $^{3,23}$, y algunos de sus componentes se han relacionado con una mayor incidencia de infartos agudos de miocardio.

De cualquier manera, nuestro estudio representa una muy pequeña serie con datos recogidos de forma retrospectiva. Cobra interés, a la luz de nuestros datos, el realizar nuevos estudios que intenten relacionar el ITB elevado con marcadores subrogados de enfermedad vascular o con eventos vasculares, así como la confirmación de nuestros datos en nuevas series de pacientes.

No contamos con una escala específica de riesgo vascular validada para población infectada por el VIH, y el ITB se ha usado sobre todo en población no infectada de mayor edad que la de las cohortes de pacientes con infección VIH publicadas hasta la fecha. El punto de corte del ITB, sobre todo cuando es elevado, en pacientes con infección VIH permanece aún pendiente de ser establecido. Necesitamos estudios que ahonden en la relación del ITB elevado con marcadores subrogados de enfermedad vascular; pero es probable que estos pacientes se puedan beneficiar de esta técnica barata, incruenta y de fácil realización para la estratificación del riesgo vascular con vistas a implementar medidas preventivas más agresivas y precoces en aquellos pacientes con valores anómalos.

\section{Referencias}

1. Weber R, Sabin CA, Friis-Moller N, Reiss P, El Sadr WM, Kirk $\mathrm{O}$, et al. Liver related deaths in persons infected with the human immunodeficiency virus: the D:A:D study. Arch Intern Med 2006; 166: 1632-41.

2. El Sadr WM, Lundgren JD, Neaton JD, Gordin F, Abrams $\mathrm{D}$, Arduino RC, et al. CD4+ count-guided interruption of antiretroviral treatment. N Engl J Med 2006; 355: 2283-96.

3. Worm SW, Sabin C, Weber R, Reiss P, El Sadr W, Dabis F et al. Risk of Myocardial Infarction in patients with HIV Infection exposed to specific individual antiretroviral drugs from the 3 major drug classes: the data collection on adverse events of anti-HIV drugs (D:A:D) study. J Infect Dis 2010; 201: 318-30.

4. Lundgren JD, Battegay M, Behrens G, De Wit S, Guaraldi G, Katlama C, et al. European AIDS Clinical Society (EACS) guidelines on the prevention and management of metabolic diseases in HIV. HIV Med. 2008; 9: 72-81.

5. Hsue PY, Lo JC, Franklin A, Bolger AF, Martin JN, Deeks SG, et al. Progression of atherosclerosis as assessed by carotid intima-media thickness in patients with HIV infection. Circulation 2004; 109: 1603-8.

6. van Vonderen MG, Smulders YM, Stehouwer CD, Danner SA, Gundy CM, Vos F, et al. Carotid intima-media thickness and arterial stiffness in HIV-infected patients: the role of HIV, antiretroviral therapy, and lipodystrophy. J Acquir Immune Defic Syndr 2009; 50: 153-61.

7. Olalla J, Salas D, De la TJ, Del Arco A, Prada JL, Martos F, et al. Ankle-brachial index in HIV infection. AIDS Res Ther 2009; 6: 6.

8. Francisci D, Giannini S, Baldelli F, Leone M, Belfiori B, Guglielmini G, et al. HIV type 1 infection, and not short-term HAART, induces endothelial dysfunction. AIDS 2009; 23: 589-96.

9. Kristoffersen US, Kofoed K, Kronborg G, Giger AK, Kjaer A, Lebech AM. Reduction in circulating markers of endothelial dysfunction in HIV-infected patients during antiretroviral therapy. HIV Med 2009; 10: 79-87.

10. Hirsch AT, Haskal ZJ, Hertzer NR, Bakal CW, Creager MA, Halperin JL, et al. ACC/AHA 2005 Practice Guidelines for the management of patients with peripheral arterial disease (lower extremity, renal, mesenteric, and abdominal aortic): a collaborative report from the American Association for Vascular Surgery/Society for Vascular Surgery, Society for Cardiovascular Angiography and Interventions, Society for Vascular Medicine and Biology, Society of Interventional Radiology, and the ACC/AHA Task Force on Practice Guidelines (Writing Committee to Develop Guidelines for the Management 
of Patients With Peripheral Arterial Disease): endorsed by the American Association of Cardiovascular and Pulmonary Rehabilitation; National Heart, Lung, and Blood Institute; Society for Vascular Nursing; TransAtlantic Inter-Society Consensus; and Vascular Disease Foundation. Circulation 2006; 113: e463-e654.

11. Fowkes FG, Murray GD, Butcher I, Heald CL, Lee RJ, Chambless LE, et al. Ankle brachial index combined with Framingham Risk Score to predict cardiovascular events and mortality: a meta-analysis. JAMA 2008; 300: 197-208.

12. Palacios R, Alonso I, Hidalgo A, Aguilar I, Sanchez MA, Valdivielso P, et al. Peripheral Arterial Disease in HIV Patients Older than 50 Years of Age. AIDS Res Hum Retroviruses 2008; 24 (8): 1043-6.

13. Anderson KM, Wilson PW, Odell PM, Kannel WB. An updated coronary risk profile. A statement for health professionals. Circulation 1991; 83: 356-62.

14. Conroy RM, Pyorala K, Fitzgerald AP, Sans S, Menotti A, De Backer G, et al. Estimation of ten-year risk of fatal cardiovascular disease in Europe: the SCORE project. Eur Heart J 2003; 24: 987-1003.

15. Executive Summary of The Third Report of The National Cholesterol Education Program (NCEP) Expert Panel on Detection, Evaluation, And Treatment of High Blood Cholesterol In Adults (Adult Treatment Panel III). JAMA. 2001; 285: 2486-97.

16. Olalla J, Salas D, Del Arco A, De la Torre J, Prada J, Machin-Hamalainen S, et al. Ankle-branch index and HIV: the role of antiretrovirals. HIV Med 2009; 10: 1-5. (17) Hiatt WR. Medical treatment of peripheral arterial disease and claudication. N Engl J Med 2001; 344: 1608-21.

17. Law MG, Friis-Moller N, El Sadr WM, Weber R, Reiss P, d'Arminio MA, et al. The use of the Framingham equation to predict myocardial infarctions in HIV-infected patients: comparison with observed events in the D:A:D Study. HIV Med 2006; 7: 218-30.

18. Schmolling Y, del Valle FJ, Pérez de Oteyza C, de Lucas A, Brasero F, Fajardo F. La medida del índice tobillo-brazo: particularmente indicada en pacientes con síndrome metabólico sin enfermedad arterial conocida. Rev Clin Esp 2008; 208 (4): 175-81.

19. Suárez C, Manzano L, Mostaza J, Cairols M, Palma JC, García I, et al. Prevalencia de enfermedad arterial periférica estimada mediante el índice tobillo-brazo en pacientes con síndrome metabólico. Estudio MERITO I. Rev Clin Esp 2007; 207 (5): 228-33.

20. Grinspoon SK, Grunfeld C, Kotler DP, Currier JS, Lundgren JD, Dube MP, et al. State of the science conference: Initiative to decrease cardiovascular risk and increase quality of care for patients living with HIV/AIDS: executive summary. Circulation 2008; 118: 198-210.

21. Gutiérrez F, Bernal E, Padilla S, Hernández I, Masia M. Relationship between ankle-brachial index and carotid intima-media thickness in HIV-infected patients. AIDS. 2008; 22: 1369-71.

22. Lang S, Mary-Krause M, Cotte L, Gilquin J, Partisani M, Simon A, et al. Impact of individual antiretroviral drugs on the risk of myocardial infarction in human immunodeficiency virus-infected patients: a case-control study nested within the French Hospital Database on HIV ANRS cohort CO4. Arch Intern Med 2010; 170: 1228-38. 